\title{
Chemotherapy combined with high-dose extended-field radiotherapy for stage I extranodal nasal-type natural killer/T-cell lymphoma
}

This article was published in the following Dove Press journal:

OncoTargets and Therapy

II October 2016

Number of times this article has been viewed

\author{
Jialin Luo* \\ Caineng Cao* \\ Yuan Zhu \\ Peng Liu \\ Luying Liu \\ $\mathrm{Ke} \mathrm{Lu}$ \\ $\mathrm{Na}$ Zhang \\ Ning Zhou
}

Department of Radiation Oncology, Zhejiang Cancer Hospital, Zhejiang

Key Laboratory of Radiation

Oncology, Hangzhou, People's

Republic of China

*These authors contributed equally to this work
Background and objective: The objective of the study was to evaluate the efficiency of chemotherapy (CT) combined with high-dose extended-field radiotherapy (RT) in stage I extranodal nasal-type natural killer/T-cell lymphoma (NKTCL).

Patients and methods: Between January 2001 and November 2010, 103 stage I extranodal nasal-type NKTCL patients were retrospectively analyzed. Of these patients, 75 patients were treated by RT plus CT and 28 patients were treated by RT alone. CT included cyclophosphamide, doxorubicin, vincristine, and prednisone (CHOP), or CHOP-like regimen.

Results: The median follow-up time was 42.6 months (range, 7.4-126.7 months). For patients in the RT alone group, the 5-year estimated progression-free survival (PFS) and overall survival (OS) rates were $67.0 \%$ and $71.4 \%$, respectively. For patients in the RT $+\mathrm{CT}$ group, the 5 -year estimated PFS and OS rates were $69.0 \%$ and $63.7 \%$, respectively. In multivariate analysis, CT was an independent factor for PFS.

Conclusion: The doxorubicin-based CT combined with high-dose extended-field RT yielded promising outcomes for stage I extranodal nasal-type NKTCL, and CT was an independent factor for PFS.

Keywords: natural killer/T-cell lymphoma, chemotherapy, radiotherapy, prognosis

\section{Introduction}

Extranodal natural killer/T-cell lymphoma (NKTCL) is relatively rare in Europe and North America but is more prevalent in South American and Asian countries. ${ }^{1,2}$ Earlystage NKTCL accounts for $70 \%-90 \%$ of cases; however, the treatment of early-stage NKTCL is controversial. ${ }^{3}$ Sequential chemotherapy (CT) and radiotherapy (RT), RT alone, or concurrent chemoradiotherapy was recommended for early-stage NKTCL in the current guidelines of the National Comprehensive Cancer Network. ${ }^{4}$

The study by $\mathrm{Li}$ et $\mathrm{al}^{5}$ indicated that only $5 \%$ patients had local relapse, $5 \%$ patients had lymph node relapse, and 17\% patients developed systemic extranodal disseminations using high-dose extended-field RT alone for stage I NKTCL.

$\mathrm{CT}$ is increasingly recognized as an indispensable treatment component for extranodal NKTCL. The aim of this study was to evaluate the efficiency of CT combined with high-dose extended-field RT in stage I extranodal nasal-type NKTCL.

\section{Materials and methods}

\section{Patients and patient workup}

This retrospective study was approved by the Independent Ethics Committee of Zhejiang Cancer Hospital. Patient consent was waived and patient records were deidentified and anonymized prior to analysis. 
The inclusion criteria were as follows: 1) histologically confirmed extranodal nasal-type NKTCL by biopsy, 2) Ann Arbor stage I disease limited to the upper aerodigestive tract without extranodal and nodal dissemination, 3) Eastern Cooperative Oncology Group (ECOG) score 0-1, 4) receiving high-dose extended-field RT (radiation dose $\geq 50 \mathrm{~Gy}$ ), 5) no previous malignancy or other concomitant malignant disease, and 6) no pregnancy or lactation.

Between January 2001 and November 2010, 103 extranodal nasal-type NKTCL patients who met all of the criteria were retrospectively analyzed. Of these, 75 patients were treated by RT $+\mathrm{CT}$ and 28 patients were treated by RT alone. The pretreatment workup included a complete history and physical examination; biochemistry analyses; complete blood cell counts; bone marrow aspiration; computed tomography scans of the chest, abdomen, and pelvis; computed tomography and/or magnetic resonance imaging of the head and neck; and dental check.

For patients with nasal NKTCL limited to the anterior part of the nasal cavity, the clinical target volume of extendedfield RT encompassed the bilateral nasal cavity, frontal ethmoid sinus, and ipsilateral maxillary sinus and, if bilateral nasal cavity was involved, the bilateral maxillary sinus was included. In case of disease close to the choanae or involving adjacent organs, the nasopharynx, paranasal sinuses (frontal and posterior ethmoid sinus if the ethmoid sinus was involved), and other adjacent organs or structures were included. Similarly, for patients with the involvement of the extranasal upper aerodigestive tract, the whole Waldeyer ring and disease extension were included. Prophylactic cervical node irradiation was routinely used for patients with Waldeyer ring NKTCL, but it was not given to patients with nasal NKTCL. A total dose of 50 Gy at 1.8-2.0 Gy per daily fraction was given to the primary tumor and residual disease after the initial 50 Gy was treated with boost irradiation 6-10 Gy.

Cyclophosphamide, doxorubicin, vincristine, and prednisone (CHOP), or CHOP-like regimen was adopted for 75 patients. The median number of CT cycles was 4 (range, 1-6) cycles. The Radiation Therapy Oncology Group and the European Organization for Research and Treatment of Cancer radiation morbidity scoring criteria were adopted for evaluating the radiation-induced toxicities. Based on the National Cancer Institute Common Terminology Criteria of Adverse Events version 3.0, CT-related adverse events were graded.

\section{Statistical analysis}

The Statistical Package for Social Sciences, version 17.0 (SPSS Inc., Chicago, IL, USA), software was used for statistical analysis. Overall survival (OS) was defined as the time from the date of initial treatment to death as a result of any causes. Progression-free survival (PFS) was defined as the time from the date of initial treatment to the first reported event, including progression, relapse, or death as a result of any causes. OS and PFS rates were computed by the KaplanMeier method, whereas survival curves were compared by the log-rank test. Chi-square and Mann-Whitney $U$-tests were used to evaluate the differences between the RT $+\mathrm{CT}$ and the RT alone groups. Using backward elimination of insignificant explanatory variables, multivariate analyses with the Cox proportional hazards model were used to test independent significance. Host factors (age and sex) were included as the covariates in all tests. All statistical tests were two-sided, and $P<0.05$ was considered to be statistically significant.

\section{Results \\ Baseline characteristics}

The clinical characteristics are listed in Table 1. Compared with patients in the RT alone group, there were more patients aged $\leq 60$ years, group $2-3,{ }^{6}$ conventional RT technique and higher radiation dose in the RT $+\mathrm{CT}$ group $(P<0.05)$.

Table I Clinical characteristics

\begin{tabular}{|c|c|c|c|}
\hline Characteristics & $\begin{array}{l}\text { RT alone group } \\
(\mathrm{N}=\mathbf{2 8})\end{array}$ & $\begin{array}{l}R T+C T \text { group } \\
(\mathrm{N}=75)\end{array}$ & $P$-value \\
\hline Male & $19(67.9)$ & $49(65.3)$ & 0.810 \\
\hline Age $\leq 60$ years & $18(64.3)$ & $62(82.7)$ & 0.046 \\
\hline \multicolumn{4}{|c|}{ Location of primary tumor } \\
\hline Nasal cavity & $27(96.4)$ & $64(85.3)$ & 0.401 \\
\hline Waldeyer ring & I (3.6) & $10(13.4)$ & \\
\hline Oral cavity & $0(0.0)$ & I (I.3) & \\
\hline PTI & $4(14.3)$ & $25(33.3)$ & 0.083 \\
\hline B symptoms & $3(10.7)$ & $19(25.3)$ & 0.107 \\
\hline Elevated LDH level & $4(14.3)$ & $25(33.3)$ & 0.119 \\
\hline \multicolumn{4}{|l|}{ ECOG score } \\
\hline 0 & $22(78.6)$ & $66(88.0)$ & 0.227 \\
\hline I & $6(21.4)$ & $9(12.0)$ & \\
\hline \multicolumn{4}{|l|}{ NKPI } \\
\hline Group I & $23(82.1)$ & $4 \mid(54.7)$ & 0.043 \\
\hline Group 2 & $3(10.7)$ & $24(32.0)$ & \\
\hline Group 3 & $2(7.1)$ & $10(13.3)$ & \\
\hline \multicolumn{4}{|c|}{ Radiotherapy technique } \\
\hline IMRT & $7(25.0)$ & $12(16.0)$ & $<0.001$ \\
\hline 3D-CRT & $6(21.4)$ & $0(0.0)$ & \\
\hline Conventional & $15(53.6)$ & $63(84.0)$ & \\
\hline \multicolumn{4}{|l|}{ Radiation dose (Gy) } \\
\hline 50 & $24(85.7)$ & $46(6 \mid .3)$ & 0.018 \\
\hline$>50, \leq 60$ & $4(14.3)$ & $29(38.7)$ & \\
\hline
\end{tabular}

Notes: PTI is defined as the presence of primary disease that extended into neighboring structures or organs or the involvement of multiple, contiguous primary sites, regardless of the stage or primary site. Data is presented as $n(\%)$.

Abbreviations: 3D-CRT, three-dimensional conformal radiotherapy; CT, chemotherapy; ECOG, Eastern Cooperative Oncology Group; IMRT, intensitymodulated radiation therapy; LDH, lactate dehydrogenase; NKPI, natural killer/T-cell lymphoma prognostic index; PTI, primary tumor invasion; RT, radiotherapy. 

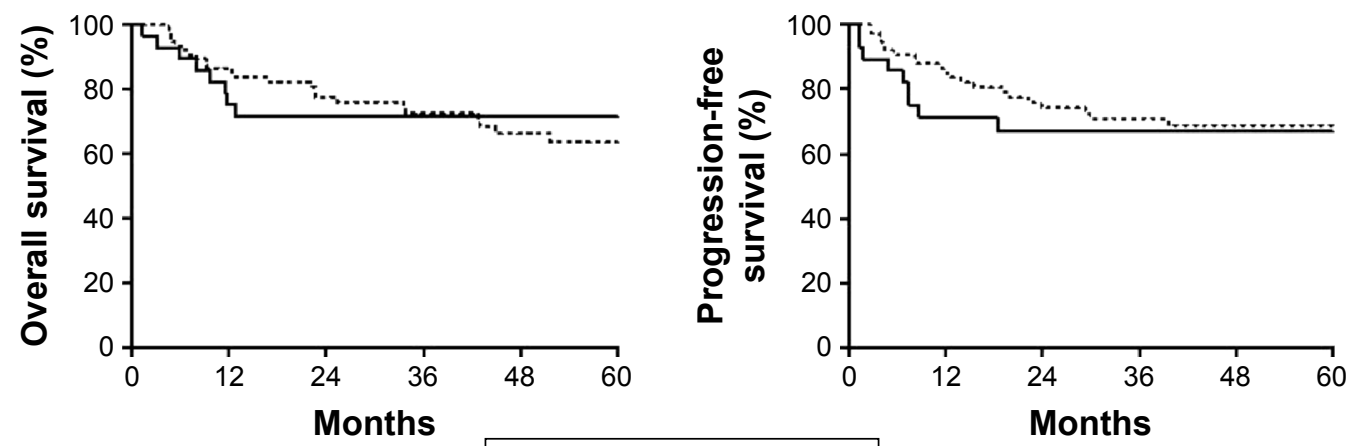

$\ldots \mathrm{RT}+\mathrm{CT}-\mathrm{RT}$ alone

Figure I Kaplan-Meier curve showing OS and PFS of patients with stage I extranodal nasal-type NKTCL treated with RT alone or RT + CT in the study Abbreviations: CT, chemotherapy; NKTCL, natural killer/T-cell lymphoma; OS, overall survival; PFS, progression-free survival; RT, radiotherapy.

No significant difference was found in terms of male, location of primary tumor, primary tumor invasion (PTI), B symptoms, elevated lactate dehydrogenase level, and ECOG score between the two groups $(P>0.05)$.

\section{Treatment outcomes}

The median follow-up time was 42.6 months (range, 7.4-126.7 months). At the last follow-up, 32 patients had died. In the RT alone group, eight (25.0\%) patients died of lymphoma. In the RT + CT group, 19 (59.4\%) patients died of lymphoma, two $(6.3 \%)$ died of severe CT-related infection, and three $(9.4 \%)$ died of concomitant illness (hepatic function failure in 1 , chronic obstructive pulmonary disease in 1 , and cerebral infarction in 1).

For patients in the RT alone group, the 5-year estimated PFS and OS rates were $67.0 \%$ and $71.4 \%$, respectively. For patients in the RT $+\mathrm{CT}$ group, the 5-year estimated PFS and OS rates were $69.0 \%$ and $63.7 \%$, respectively. No statistically significant difference was observed in terms of PFS and OS between the RT alone and the RT + CT groups (Figure 1). No statistically significant difference was observed in PFS (5-year estimated PFS: $72.6 \%$ vs 59.0\%, $P=0.275$ ) and OS (5-year estimated OS: $68.7 \%$ vs $54.4 \%$, $P=0.311$ ) between the patients receiving 50 Gy and in the patients receiving $>50 \mathrm{~Gy}$.

\section{Toxicity}

In the RT alone group, most of patients had Grade $0-2$ acute toxicities and only one patient had acute Grade 3 mucositis. In the $\mathrm{RT}+\mathrm{CT}$ group excluding two patients who died of severe CT-related infection, the incidence of $\geq$ Grade 3 acute mucositis, nausea/vomit, leukopenia, and thrombocytopenia was $1.4 \%, 9.6 \%, 34.2 \%$, and $6.8 \%$, respectively. Severe late complication ( $\geq$ Grade 3 ) or second malignancy was not observed in all patients.

\section{Prognostic factors}

The values of potential prognostic factors including sex, age, location of primary tumor, PTI, ECOG score, natural killer/Tcell lymphoma prognostic index, ${ }^{6}{ }^{\mathrm{RT}}$ technique, radiation dose and CT on predicting PFS, and OS were evaluated. The outcomes are shown in Table 2. In multivariate analysis, CT was an independent factor for PFS.

\section{Discussion}

This study assessed the treatment outcomes of stage I extranodal nasal-type NKTCL treated by high-dose extended-field RT. Patients with stage I extranodal nasal-type NKTCL were classified into RT + CT or RT alone groups. We have demonstrated that CT was an independent factor for PFS.

Various treatment options, RT volumes and doses, and CT regimens were adopted for NKTCL at different institutions. ${ }^{3}$ Traditionally, early-stage aggressive T-cell lymphoma is treated by doxorubicin-based CT with or without RT based on the evidences of diffuse large B-cell lymphoma. However, NKTCL is resistant to CT. The outcomes of

Table 2 Multivariate analysis of prognostic factors for patients with stage I extranodal nasal-type NKTCL

\begin{tabular}{lllll}
\hline Endpoint & Items & HR & $\mathbf{9 5 \%} \mathbf{~ C l}$ & P-value \\
\hline PFS & NKPI & & & \\
& Group I & 1.0 & & \\
& Group 2 & 5.723 & $2.296-14.263$ & $<0.001$ \\
& Group 3 & 2.501 & $0.617-10.136$ & 0.199 \\
& Chemotherapy & & & \\
& No & 1.0 & & \\
OS & Yes & 0.292 & $0.113-0.753$ & 0.011 \\
& NKPI & & & \\
& Group I & 1.0 & & \\
& Group 2 & 2.638 & $1.191-5.842$ & 0.017 \\
& Group 3 & 2.751 & $0.884-8.556$ & 0.081 \\
\hline
\end{tabular}

Abbreviations: NKPI, natural killer/T-cell lymphoma prognostic index; NKTCL, natural killer/T-cell lymphoma; OS, overall survival; PFS, progression-free survival; $\mathrm{HR}$, hazard ratio; $\mathrm{Cl}$, confidence interval. 
early-stage NKTCL treated by CT alone were poor (5-year OS, $28.6 \%-35 \%))^{7,8}$ RT is the backbone of curative intent for stage I extranodal nasal-type NKTCL, ${ }^{3}$ most of the previous studies did not show the benefit of adding CT to RT in stage I extranodal nasal-type NKTCL., ${ }^{9}, 10$ With regard to the low efficacy of CT, the efficiency of CT combined with high-dose extended-field RT for stage I extranodal nasaltype NKTCL was evaluated, and it was found that CT was an independent factor for PFS.

Recently, an L-asparaginase-containing CT regimen has been evaluated in advanced, refractory, and relapsed NKTCL, with a complete response rate and overall response rate of $45 \%-56 \%$ and $79 \%-78 \%$, respectively. ${ }^{11,12}$ Two Phase II prospective studies indicated that L-asparaginasebased CT combined with RT yielded promising outcomes for early-stage nasal-type NKTCL, with a 2-year PFS and OS rate of $>80 \% .{ }^{13,14}$ The prognosis of stage I extranodal nasal-type NKTCL may be additionally improved by the adoption of new effective CT plus high-dose extended-field RT.

The result of our study to detect small differences in outcomes was limited for the small number of patients, the retrospective review, and the heterogeneity of patients. Given the rarity of NKTCL, the inclusion of patients in a rare cancer registry or multicentre study would provide the best opportunity to accurately evaluate the role of CT.

\section{Conclusion}

The doxorubicin-based CT combined with high-dose extended-field RT yielded promising outcomes for stage I extranodal nasal-type NKTCL, and CT was an independent factor for PFS.

\section{Disclosure}

The authors report no conflicts of interest in this work.

\section{References}

1. Chim CS, Ma SY, Au WY, et al. Primary nasal natural killer cell lymphoma: long-term treatment outcome and relationship with the International Prognostic Index. Blood. 2004;103:216-221.

2. Vose J, Armitage J, Weisenburger D. International peripheral T-cell and natural killer/T-cell lymphoma study: pathology findings and clinical outcomes. J Clin Oncol. 2008;26:4124-4130.

3. Yang Y, Zhu Y, Cao JZ, et al. Risk-adapted therapy for early-stage extranodal nasal-type NK/T-cell lymphoma: analysis from a multicenter study. Blood. 2015;126:1424-1432.

4. National Comprehensive Cancer Network Guidelines: non-Hodgkin's lymphomas. Available at: http://www.nccn.org/professionals/ physician_gls/f_guidelines.asp\#nhl. Accessed June 27, 2015.

5. Li YX, Wang H, Jin J, et al. Radiotherapy alone with curative intent in patients with stage I extranodal nasal-type NK/T-cell lymphoma. Int $J$ Radiat Oncol Biol Phys. 2012;82:1809-1815.

6. Lee J, Suh C, Park YH, et al. Extranodal natural killer T-cell lymphoma, nasal-type: a prognostic model from a retrospective multicenter study. J Clin Oncol. 2006;24:612-618.

7. Li CC, Tien HF, Tang JL, et al. Treatment outcome and pattern of failure in 77 patients with sinonasal natural killer/T-cell or T-cell lymphoma. Cancer. 2004;100:366-375.

8. You JY, Chi KH, Yang MH, et al. Radiation therapy versus chemotherapy as initial treatment for localized nasal natural killer (NK)/ T-cell lymphoma: a single institute survey in Taiwan. Ann Oncol. 2004;15:618-625

9. Li YX, Yao B, Jin J, et al. Radiotherapy as primary treatment for stage IE and IIE nasal natural killer/T-cell lymphoma. J Clin Oncol. 2006;24:181-189.

10. Ma HH, Qian LT, Pan HF, et al. Treatment outcome of radiotherapy alone versus radiochemotherapy in early stage nasal natural killer/ T-cell lymphoma. Med Oncol. 2010;27:798-806.

11. Yamaguchi M, Kwong YL, Kim WS, et al. Phase II study of SMILE chemotherapy for newly diagnosed stage IV, relapsed, or refractory extranodal natural killer (NK)/T-cell lymphoma, nasal type: the NKCell Tumor Study Group study. J Clin Oncol. 2011;29:4410-4416.

12. Kwong YL, Kim WS, Lim ST, et al. SMILE for natural killer/T-cell lymphoma: analysis of safety and efficacy from the Asia Lymphoma Study Group. Blood. 2012;120:2973-2980.

13. Wang L, Wang ZH, Chen XQ, et al. First-line combination of gemcitabine, oxaliplatin, and L-asparaginase (GELOX) followed by involvedfield radiation therapy for patients with stage IE/IIE extranodal natural killer/T-cell lymphoma. Cancer. 2013;119:348-355.

14. Jiang M, Zhang H, Jiang Y, et al. Phase 2 trial of "sandwich" L-asparaginase, vincristine, and prednisone chemotherapy with radiotherapy in newly diagnosed, stage IE to IIE, nasal type, extranodal natural killer/T-cell lymphoma. Cancer. 2012;118:3294-3301.
OncoTargets and Therapy

\section{Publish your work in this journal}

OncoTargets and Therapy is an international, peer-reviewed, open access journal focusing on the pathological basis of all cancers, potential targets for therapy and treatment protocols employed to improve the management of cancer patients. The journal also focuses on the impact of management programs and new therapeutic agents and protocols on
Dovepress

patient perspectives such as quality of life, adherence and satisfaction. The manuscript management system is completely online and includes a very quick and fair peer-review system, which is all easy to use. Visit http://www.dovepress.com/testimonials.php to read real quotes from published authors. 